\title{
What is known about consumer nutrition environments in Australia?
}

\author{
C.E. Pulker ${ }^{1}$, L. Thornton ${ }^{2}$ and G. Trapp ${ }^{3}$ \\ ${ }^{1}$ School of Public Health, Curtin University, Kent Street, GPO Box U1987, Perth 6845, Western Australia, Australia \\ ${ }^{2}$ Centre for Physical Activity and Nutrition Research, School of Exercise and Nutrition Sciences, Deakin University, \\ Melbourne Burwood Campus, 221 Burwood Highway, Burwood, Melbourne 3125, Victoria, Australia and \\ ${ }^{3}$ Telethon Kids Institute, The University of Western Australia, 35 Stirling Highway, Perth 6009, Western Australia, \\ Australia
}

A new and emerging area of food environments research is investigating what consumers encounter within and around a food outlet, referred to as the 'consumer nutrition environment ${ }^{\text {'(1) }}$. Although several systematic reviews have summarised associations between consumer nutrition environments and health, none have addressed all aspects of the consumer nutrition environment, and few of the studies included have been conducted outside of the United States of America. The main objective of this systematic scoping review was to summarise existing peer-reviewed Australian studies that have examined consumer nutrition environments, identify knowledge gaps, and provide recommendations for future research.

The five step scoping review protocol by Arksey and O'Malley was used ${ }^{(2)}$. Ovid Medline and CINAHL were searched for literature relating to the concepts of 'food and health', 'food retail outlet', 'consumer nutrition environments', and limited to Australia. Consumer nutrition environment domains which potentially influence food purchasing and eating patterns were identified, and findings relating to these aspects were recorded. Domains of the consumer nutrition environment include: Products i.e., product availability and quality, product assortment, product and packaging design, and provision of supermarket own brands; Price i.e., the price of healthy and unhealthy foods, price sensitivity and elasticity, and price promotions; Placement i.e., in-store location of products, or position and prominence of shelf-location of products; and Promotions i.e., health messages, promotions targeting children, and other methods including signage ${ }^{(3)}$.

Fifty-five studies were included. Many studies were conducted in capital cities, on the eastern seaboard of Australia, in supermarkets; over half were from 2011 onwards. Some investigations of remote community stores and fast food outlets were also included, as were studies spanning a range of outlet types. The most studied domain was product (37/55), followed by price (24/55), promotion (13/55), and placement (5/55). Analysis identified forty-seven themes relating to healthy and unhealthy foods. Most common were: socioeconomic patterning of healthy food prices (10/55); impact of food outlet type on prices of healthy food (6/55); classification of foods as healthy (5/55); and nutritional quality of unhealthy child-orientated products (5/55).

This scoping review revealed what is currently known about Australian consumer nutrition environments. As an emerging field, there are a large number of gaps in knowledge. No studies have addressed all four domains, reporting only selected aspects. It is important to build a more comprehensive understanding of the food environments Australians face when selecting and purchasing food. This will assist public health professionals to develop policies and practices to improve placement, promotion, pricing, and availability of healthy foods at a local, state or territory, and national level.

1. Glanz K, Sallis JF, Saelens BE, Frank LD (2005) Healthy nutrition environments: concepts and measures. Am J Health Promot 19(5), 330-333.

2. Arksey H \& O'Malley L (2005) Scoping studies: towards a methodological framework. International Journal of Social Research Methodology. 8(1), 19-32.

3. Glanz K, Bader MD \& Iyer S (2012) Retail grocery store marketing strategies and obesity: an integrative review. Am J Prev Med 42(5), 503-512. 